COLO-HEP-279

\title{
ISING MODEL COUPLED TO THREE-DIMENSIONAL QUANTUM GRAVITY
}

\author{
C.F. Baillie \\ Physics Department \\ University of Colorado \\ Boulder, CO 80309
}

July 12, 2018

\begin{abstract}
We have performed Monte Carlo simulations of the Ising model coupled to threedimensional quantum gravity based on a summation over dynamical triangulations. These were done both in the microcanonical ensemble, with the number of points in the triangulation and the number of Ising spins fixed, and in the grand canonical ensemble. We have investigated the two possible cases of the spins living on the vertices of the triangulation ("direct" case) and the spins living in the middle of the tetrahedra ("dual" case). We observed phase transitions which are probably second order, and found that the dual implementation more effectively couples the spins to the quantum gravity.
\end{abstract}




\section{Introduction}

Following recent Monte Carlo simulations of spin models coupled to two-dimensional quantum gravity [1] and of pure three-dimensional quantum gravity [2] we have performed some small exploratory simulations of the Ising model coupled to three-dimensional quantum gravity. This is the simplest model of matter coupled to quantum gravity in three dimensions, and provides a step on the way to simulating the physical case of matter coupled to fourdimensional quantum gravity.

The continuum Einstein action for 3-dimensional gravity is

$$
S_{E}=\int d^{3} \xi \sqrt{g}\left(\lambda-\frac{R}{2 \pi G}\right)
$$

where $\lambda$ is the cosmological constant and $G$ is Newton's constant. When a dynamical triangulation (with $N_{0}$ vertices, $N_{1}$ links, $N_{2}$ triangles and $N_{3}$ tetrahedra) is used to regularize the functional integration over metrics the two terms in (1) discretize as follows:

$$
\begin{gathered}
\int d^{3} \xi \sqrt{g} \rightarrow N_{3} \\
\int d^{3} \xi \sqrt{g} R \rightarrow \sum_{l}(c-n(l)),
\end{gathered}
$$

where $n(l)$ is the number of tetrahedra which share the link $l$, and $c=2 \pi / \alpha$, with $\alpha=$ $\arccos \left(\frac{1}{3}\right)$, is a number which ensures that $R$ is zero for flat space. Since each tetrahedron is comprised of six links, $\sum_{l} n(l)=6 N_{3}$ and (3) can be written as $c N_{1}-6 N_{3}$. Hence the discrete version of (四) is

$$
S=\lambda N_{3}-\frac{1}{2 \pi G}\left(c N_{1}-6 N_{3}\right)
$$

Now since we have the following relations for a three-dimensional triangulation (or "threedimensional simplicial manifold")

$$
\begin{gathered}
N_{3}-N_{2}+N_{1}-N_{0}=0 \\
N_{2}=2 N_{3}
\end{gathered}
$$

(first is Euler's relation; second is because each triangle is common to two tetrahedra), we can change to the more convenient variables $N_{0}, N_{3}$ and write

$$
S=\lambda_{3} N_{3}-\lambda_{0} N_{0}
$$

with $\lambda_{3}=\left(\lambda+\frac{6-c}{2 \pi G}\right)$ and $\lambda_{0}=\frac{c}{2 \pi G}$. Therefore the grand canonical partition function for quantum gravity in three dimensions based on a summation over dynamical triangulations $T$ with $N_{3}$ tetrahedra and $N_{0}$ vertices is

$$
Z\left(\lambda_{3}, \lambda_{0}\right)=\int d N_{3} d N_{0} Z_{N_{3}, N_{0}} e^{-\lambda_{3} N_{3}+\lambda_{0} N_{0}}
$$


where

$$
Z_{N_{3}, N_{0}}=\sum_{T\left(N_{3}, N_{0}\right)} \rho(T)
$$

is the micro-canonical partition function in which $N_{3}, N_{0}$ are fixed, and $\rho(T)$ is a suitable weight for each triangulation $T$. One usually appeals to universality and sets $\rho(T)=1$.

In order to perform a Monte Carlo simulation of either of these partition functions for pure three-dimensional quantum gravity one must define a set of "moves" which ergodically sum over all possible triangulations. In two dimensions this is straight-forward since one move suffices for each case: for the grand canonical partition function one uses the so-called "split-join" move, and for the micro-canonical partition function the "flip". Unfortunately things are not as simple in three dimensions - there is no known set of moves which is ergodic in the micro-canonical ensemble; however two moves exist for the grand canonical case: the so-called "Alexander move" which is a generalization of the two-dimensional split-join move changing one tetrahedron into four tetrahedra (or vice versa), and a generalization of the "flip" taking two tetrahedra into three tetrahedra (or vice versa). For more details and illustrations of these moves see [2].

If we now couple the Ising model to this discretization of three-dimensional quantum gravity the action (17) becomes

$$
S_{I}=\lambda_{3} N_{3}-\lambda_{0} N_{0}+\beta \sum_{i, j} S_{i j}\left(1-\sigma_{i} \sigma_{j}\right)
$$

where $S_{i j}$ is the connectivity matrix of the triangulation, $\sigma_{i}$ are the Ising spins taking values +1 or -1 , and $\beta$ is the inverse temperature. There are two possible ways in which to add the Ising spins to the dynamical triangulation: they can either live on the vertices in which case there will be $N_{0}$ spins or they can live in the tetrahedra giving $N_{3}$ spins. We shall refer to the former as the "direct" case and the latter as the "dual". For the direct implementation there are $N_{1}=N_{0}+N_{3}$ links between the spins so $S_{i j}$ will contain this number of unit entries; for the dual case there are $N_{2}=2 N_{3}$ interactions, and hence non-zeros in $S_{i j}$. Note that we have arranged the normalization such that at zero temperature, i.e. $\beta=\infty$, all the Ising spins line up and their contribution to $S_{I}$ vanishes. At $\beta=0$ we recover pure quantum gravity.

As for the pure three-dimensional quantum gravity case, there are two possible partition functions: grand canonical and micro-canonical. For the grand canonical ensemble, one must create and destroy Ising spins when either vertices in the direct case or tetrahedra in the dual case are created and destroyed. Destruction of Ising spins is trivial - they are simply removed - however creation is rather more subtle - the values of the new spins should be picked randomly to avoid any bias. Our simulation of the grand canonical Ising plus three-dimensional quantum gravity uses the same methods as were used in all the pure three-dimensional quantum gravity simulations, see [2] for details.

As a step on the way to the grand canonical simulation, we have chosen to simulate the micro-canonical ensemble. To do this $N_{3}, N_{0}$ and the number of Ising spins must be fixed. Therefore we use only the flip move to update the triangulation, and we use it in pair-wise fashion - firstly changing two tetrahedra to three tetrahedra then immediately 
after (somewhere else in the triangulation) changing three tetrahedra to two tetrahedra. As explained above this is not known to be ergodic for the pure three-dimensional quantum gravity case, so we cannot assume it to be when we couple in Ising spins. However it is an easier simulation to do and may provide an approximation to the more realistic grand canonical case (this is somewhat like simulating quenched QCD as an approximation to full QCD in lattice gauge theory).

For both the grand canonical and micro-canonical simulations, in addition to updating the triangulation, we must update the Ising spins. To do this we make use of cluster algorithms, introduced in [3], which are very effective in the vicinity of phase transitions, where spins tend to form large clusters. However, away from the transition, the standard Metropolis method is more practical. Therefore, we used both algorithms to update the Ising spins, doing one pass of each per update step. We actually used Wolff's version of the cluster algorithm [四], as it is faster than Swendsen-Wang's [3].

\section{Micro-canonical}

Physically the vacuum in four-dimensional gravity is almost flat, therefore in our microcanonical simulations of three-dimensional quantum gravity we pick $N_{3}, N_{0}$ so that the average curvature of the triangulation

$$
\bar{R}=\frac{2 \pi\left(N_{3}+N_{0}\right)-6 \alpha N_{3}}{N_{3}}
$$

is as small as possible; which in turn implies that $\frac{N_{0}}{N_{3}} \approx .1755$. This leads to the following values of $N_{0}, N_{3}: 69,393 ; 154,878$ and 325,1852 . If $n_{3}^{i}$ is the number of tetrahedra meeting at the vertex $i$ then, since each tetrahedron has four vertices, $\sum_{i} n_{3}^{i}=4 N_{3}$, and therefore $<n_{3}>=4 \frac{N_{3}}{N_{0}} \approx 22.8$. Thus each vertex is shared by roughly 23 tetrahedra. This means that for the direct implementation of the Ising spins on the triangulation, each spin will have on average 23 neighbors, whereas for the dual implementation each spin will have only four neighbors (the number of faces of a tetrahedron). Therefore we can expect a much stronger correlation of spins for a given $\beta$ for the direct case compared to the dual case, which causes the significantly stronger phase transition to occur at a lower critical value of $\beta$. We shall first present our results for the direct case and then for the dual case. In both cases we measured the standard thermodynamic energy $E$ and magnetization $M$ for the Ising model, the acceptance rate of the Metropolis algorithm $a_{M}$, the acceptance rate for the flip moves $a_{f}$, and the fractal dimension of the spin clusters constructed by the Wolff algorithm.

For the direct case the number of Ising spins is $N_{0}=69,154$ and 325. We ran simulations at the following 18 values of $\beta$ : 0.01, 0.02, 0.03, 0.0325, 0.035, 0.0375, 0.04, 0.0425, 0.045, 0.0475, $0.05,0.06,0.07,0.08,0.09,0.1,0.2,0.3$. The results for $E, M, a_{M}, a_{f}$ for the $N_{0}=325$ simulation are listed in Table 1. 


\begin{tabular}{|c|c|c|c|c|}
\hline$\beta$ & $E$ & $M$ & $a_{M}$ & $a_{f}$ \\
\hline 0.01 & $0.99(6)$ & $0.047(1)$ & $0.973(1)$ & $0.995(1)$ \\
0.02 & $0.98(3)$ & $0.054(1)$ & $0.943(1)$ & $0.990(1)$ \\
0.03 & $0.95(2)$ & $0.077(1)$ & $0.904(1)$ & $0.985(1)$ \\
0.0325 & $0.94(2)$ & $0.083(1)$ & $0.890(1)$ & $0.984(1)$ \\
0.0350 & $0.92(2)$ & $0.098(2)$ & $0.874(1)$ & $0.983(1)$ \\
0.0375 & $0.90(2)$ & $0.124(2)$ & $0.852(1)$ & $0.982(1)$ \\
0.04 & $0.86(3)$ & $0.154(5)$ & $0.824(1)$ & $0.980(1)$ \\
0.0425 & $0.82(1)$ & $0.187(2)$ & $0.788(1)$ & $0.980(1)$ \\
0.0450 & $0.75(1)$ & $0.236(1)$ & $0.751(1)$ & $0.979(1)$ \\
0.0475 & $0.71(1)$ & $0.266(1)$ & $0.715(1)$ & $0.978(1)$ \\
0.05 & $0.66(1)$ & $0.301(2)$ & $0.683(1)$ & $0.978(1)$ \\
0.06 & $0.53(1)$ & $0.384(1)$ & $0.592(1)$ & $0.976(1)$ \\
0.07 & $0.45(1)$ & $0.444(2)$ & $0.529(1)$ & $0.975(1)$ \\
0.08 & $0.40(1)$ & $0.487(1)$ & $0.480(1)$ & $0.974(1)$ \\
0.09 & $0.35(1)$ & $0.529(1)$ & $0.440(1)$ & $0.973(1)$ \\
0.10 & $0.32(1)$ & $0.566(1)$ & $0.403(1)$ & $0.973(1)$ \\
0.20 & $0.11(1)$ & $0.840(1)$ & $0.160(1)$ & $0.980(1)$ \\
0.30 & $0.04(1)$ & $0.930(1)$ & $0.067(1)$ & $0.988(1)$ \\
\hline
\end{tabular}

Table 1: Measured values of energy $E$, magnetization $M$, Metropolis acceptance $a_{M}$ and flip acceptance $a_{f}$ from $N_{0}=325$ direct simulation.

By numerically differentiating the energy and magnetization we obtain the specific heat and susceptibility shown for all $N_{0}$ in Figs. 1 and 2 respectively. Using a cubic spline approximation we estimate the positions $C_{\max }$ and $\chi_{\max }$ of the maximum of the peaks to obtain the critical inverse temperatures $\beta_{c}$ for each $N_{0}$ listed in Table 2.

\begin{tabular}{|c|c|c|}
\hline$N_{0}$ & $\beta_{c}$ for $C_{\max }$ & $\beta_{c}$ for $\chi_{\max }$ \\
\hline 69 & $0.071(1)$ & $0.069(1)$ \\
154 & $0.056(1)$ & $0.055(1)$ \\
325 & $0.050(2)$ & $0.046(2)$ \\
\hline
\end{tabular}

Table 2: Position of peaks in specific heat and susceptibility for each value of $N_{0}$ from direct simulation.

We could attempt to fit to the specific heat and susceptibility peaks in order to extract exponents and/or we could try finite-size scaling analysis but as these are exploratory simulations of small systems the results would not be very impressive or reliable. Therefore we 
leave this for future calculations. Here we can say only that there is a phase transition, at approximately $\beta_{c}=0.048(2)$ for $N_{0}=325$, which is probably second order since the peaks in $C$ and $\chi$ increase with system size (first order is ruled out as there are no discontinuites in $E$ and $M$ ). Unfortunately without the critical exponents it is not possible to extrapolate the $\beta_{c}$ values to $N_{0}=\infty$.

Turning to the acceptance rates we see that the Metropolis acceptance falls off as $\beta$ increases as expected - the spins become frozen as the temperature falls. However the flip acceptance rate does not change very much from its initial value of 1 at $\beta=0$ (where the spins are random so do not systematically effect the triangulation). In other words the Ising spins do not seem to interact very strongly with three-dimensional quantum gravity when they are implemented directly - i.e. live on the vertices of the triangulation. Numerically this is obviously due to the fact that during the flip move only one out of on average 23 Ising spins at each vertex is reconnected so the energy change is relatively small.

Now we turn to the results for the dual case, where the number of Ising spins is $N_{3}=$ 393,878 and 1852. Simulations were done at 12 values of $\beta$ : $0.1,0.2,0.3,0.325,0.35,0.375$, $0.4,0.425,0.45,0.475,0.5,0.6$; the results from the $N_{3}=878$ simulation are in Table 3 .

\begin{tabular}{|c|c|c|c|c|}
\hline$\beta$ & $E$ & $M$ & $a_{M}$ & $a_{f}$ \\
\hline 0.1 & $0.894(7)$ & $0.033(1)$ & $0.846(1)$ & $0.782(1)$ \\
0.2 & $0.774(3)$ & $0.044(1)$ & $0.682(1)$ & $0.569(1)$ \\
0.3 & $0.639(2)$ & $0.068(2)$ & $0.513(1)$ & $0.376(1)$ \\
0.325 & $0.599(2)$ & $0.084(2)$ & $0.470(8)$ & $0.333(7)$ \\
0.350 & $0.560(1)$ & $0.105(2)$ & $0.427(9)$ & $0.291(7)$ \\
0.375 & $0.516(1)$ & $0.148(3)$ & $0.381(9)$ & $0.250(8)$ \\
0.4 & $0.456(1)$ & $0.254(5)$ & $0.327(1)$ & $0.206(1)$ \\
0.425 & $0.352(1)$ & $0.539(5)$ & $0.240(7)$ & $0.147(9)$ \\
0.450 & $0.255(1)$ & $0.728(2)$ & $0.168(9)$ & $0.106(5)$ \\
0.475 & $0.191(1)$ & $0.818(2)$ & $0.121(8)$ & $0.083(3)$ \\
0.5 & $0.146(1)$ & $0.872(1)$ & $0.088(1)$ & $0.068(1)$ \\
0.6 & $0.052(1)$ & $0.960(1)$ & $0.029(3)$ & $0.043(1)$ \\
\hline
\end{tabular}

Table 3: Measured values of energy $E$, magnetization $M$, Metropolis acceptance $a_{M}$ and flip acceptance $a_{f}$ from $N_{3}=878$ dual simulation.

We show the specific heat and susceptibility, obtained from numerical differentiation, for $N_{3}=393,878$ (we do not have enough data for $N_{3}=1852$ ), in Figs. 3 and 4 respectively. And we estimate the position of the maximum of the peaks - Table 4 .

\begin{tabular}{|c|c|c|}
\hline$N_{3}$ & $\beta_{c}$ for $C_{\max }$ & $\beta_{c}$ for $\chi_{\max }$ \\
\hline 393 & $0.444(1)$ & $0.445(1)$ \\
878 & $0.421(2)$ & $0.416(2)$ \\
\hline
\end{tabular}


Table 4: Position of peaks in specific heat and susceptibility for two values of $N_{3}$ from dual simulation.

Again it is not worth attempting to fit to this data to extract critical exponents. For now we can say that we have seen a second order phase transition at approximately $\beta_{c}=0.418(2)$ for $N_{3}=878$.

Thus for the dual case, the Ising spins significantly affect the triangulation - the acceptance rate for the flip move drops from near 1 at low $\beta$ to almost 0 as $\beta$ increases above $\beta_{c}$. Thus matter in the form of Ising spins interacts strongly with quantum gravity when it is coupled to it through the dual of the triangulation - i.e. when the spins live in the tetrahedra. If we compare the direct and dual cases we see that for the former the peaks in $C$ and $\chi$ are much larger as expected. Interestingly the critical value of $\beta$ for the dual case in which each spin is connected to four neighbors $\left(\beta_{c}=0.418(2)\right)$ is closer to the value $(0.221652(3)$ [5]) for the standard 3D Ising model which has coordination number six, than is the value $(0.048(2))$ from the direct case with its average coordination number of 23 .

Finally, we look at the fractal dimension of the clusters constructed by the Wolff algorithm. This is interesting because, as discussed by Meo, Heermann and Binder [6], these clusters are actually the real, physical clusters in the system. For a standard Ising model simulated in $D$ dimensions, the fractal dimension $d$ is given by $d=D-\beta / \nu$ which is approximately 2.5 for $D=3$ [7]. In the simulations we stored the sizes and diameters $\mathbb{\text { D }}$ of the Ising clusters. The $\log -\log$ plots of the average cluster size versus it's diameter along with the best fits for $d$, for both the direct and dual simulations, are shown in Fig. 5. For the direct case we get a value of $d=2.7(2)$ close to the standard 3D Ising model value, for the dual case it is a little smaller $(2.0(1))$. These values should be considered as preliminary given the small triangulations used - the maximum sized clusters which fit on the direct and dual triangulations are only about 50 and 100 sites respectively.

\section{Grand canonical}

Armed with the knowledge that the dual implementation of the Ising spins on the triangulation more effectively couples them to three-dimensional quantum gravity in the microcanonical ensemble, we performed a modest simulation of the dual case in the grand canonical ensemble. To do this one first picks likely values of the parameters $\lambda_{3}, \lambda_{0}$ plus widths $\Delta \lambda_{3}, \Delta \lambda_{0}$ then lets the Monte Carlo simulation vary the former within the latter and find the actual values of $\lambda_{3}, \lambda_{0}$. We started with $\lambda_{3}=1, \lambda_{0}=0, \Delta \lambda_{3}=\Delta \lambda_{0}=0.25$ and ran the first simulation with $N_{3}=393$ at $\beta=0.1,0.2,0.3,0.35,0.4,0.45,0.5$. This resulted in the predictions for $\lambda_{3}, \lambda_{0}$ listed in columns 2 and 3 respectively in Table 5 . Then we used these predictions as start values for the second simulation with $N_{3}=878$ resulting in the further predictions listed in columns 4 and 5 . Also listed in Table 5 , in the " $\beta=0$ " row, are the values of $\lambda_{3}, \lambda_{0}$ from simulations of pure three-dimensional quantum gravity on the same-sized triangulations [2]. In these pure quantum gravity simulations a phase transition

\footnotetext{
${ }^{1}$ By diameter we mean the distance from the random starting point used in construction of the cluster to the most remote point on the cluster border; on average this should be proportional to the gyration radius.
} 
was found at $\lambda_{0} \approx 0.61$; for $\lambda_{0}<0.61$ we are in a "strong gravity" phase (Newton's constant $G \sim \frac{1}{\lambda_{0}}$ is large) where the fractal or Hausdorff dimension $d_{H}$ of the triangulation becomes large. (Note $d_{H}$ should not be confused with $d$, the fractal dimension of the Ising spin clusters.) In Fig. 6 we see a roughly linear relationship between the $\lambda_{3}, \lambda_{0}$ values and $\beta$ with no dramatic change at the Ising model phase transition around $\beta_{c}=0.4$. The Ising model reduces the values of both $\lambda_{3}, \lambda_{0}$ and therefore has the effect of reducing the cosmological constant and making already strong gravity stronger. Perhaps this makes sense - matter does couple positively to gravity after all, and the cosmological constant is essentially zero in the real universe. However, it is also apparent that there are large finite-size corrections which tend to increase $\lambda_{3}$ and decrease $\lambda_{0}$. Therefore when large simulations are done it may turn out that $\lambda_{0}$ remains at its pure quantum gravity value despite the addition of matter in the form of Ising spins, whereas $\lambda_{3}$ is significantly reduced, perhaps to zero.

\begin{tabular}{|c|c|c|c|c|}
\hline$\beta$ & $\lambda_{3}(393)$ & $\lambda_{0}(393)$ & $\lambda_{3}(878)$ & $\lambda_{0}(878)$ \\
\hline 0 & 1.38 & 0.24 & 1.40 & 0.36 \\
0.10 & $1.25(1)$ & $0.19(1)$ & $1.202(8)$ & $0.360(8)$ \\
0.20 & $1.16(1)$ & $0.15(1)$ & $1.101(8)$ & $0.337(8)$ \\
0.30 & $1.09(1)$ & $0.12(1)$ & $1.017(8)$ & $0.319(8)$ \\
0.35 & $1.04(1)$ & $0.11(1)$ & $0.961(8)$ & $0.314(9)$ \\
0.40 & $1.03(1)$ & $0.10(1)$ & $0.945(8)$ & $0.308(9)$ \\
0.45 & $0.97(1)$ & $0.06(1)$ & $0.900(8)$ & $0.284(8)$ \\
0.50 & $0.96(1)$ & $0.05(1)$ & $0.875(8)$ & $0.253(8)$ \\
\hline
\end{tabular}

Table 5: Predicted values of $\lambda_{3}, \lambda_{0}$ from grand canonical dual simulations with $N_{3}=393$ and 878 .

In addition to $E, M, a_{M}, a_{f}$ we also measured the average curvature of the triangulation. In the strong gravity regime for pure quantum gravity this is found to be negative [2] and indeed we find this here. The values of $E, M$ and $a_{M}$ for the grand canonical simulation are consistent with those for the micro-canonical; therefore, despite doubts about its ergodicity, the micro-canonical simulation does provide a good approximation to the grand canonical. The values of $a_{f}$ are substantially reduced because now this quantity includes the acceptance rate for the Alexander moves as well as the flip moves, and the former are greatly suppressed by the relatively large change in energy brought about by the creation and destruction of (in this case three) Ising spins.

The fractal dimension of the clusters constructed by the Wolff algorithm, remains close to that found for the micro-canonical simulation, namely a little less that the value for the standard 3D Ising model. This is probably due to the fact that the underlying triangulation has a large Hausdorff dimension, therefore clusters of spins built upon it grow more slowly than those built with an underlying 3D lattice. 


\begin{tabular}{|c|c|c|c|c|c|}
\hline$\beta$ & $E$ & $M$ & $a_{M}$ & $a_{f}$ & $\bar{R}$ \\
\hline 0.10 & $0.903(1)$ & $0.034(1)$ & $0.85(3)$ & $0.107(12)$ & $-0.333(16)$ \\
0.20 & $0.784(1)$ & $0.045(1)$ & $0.69(3)$ & $0.105(12)$ & $-0.383(16)$ \\
0.30 & $0.655(1)$ & $0.068(1)$ & $0.54(3)$ & $0.097(12)$ & $-0.409(17)$ \\
0.35 & $0.585(1)$ & $0.099(2)$ & $0.47(4)$ & $0.087(11)$ & $-0.414(17)$ \\
0.40 & $0.494(1)$ & $0.221(4)$ & $0.38(5)$ & $0.077(12)$ & $-0.411(18)$ \\
0.45 & $0.270(1)$ & $0.713(1)$ & $0.20(5)$ & $0.054(11)$ & $-0.448(17)$ \\
0.50 & $0.147(1)$ & $0.871(1)$ & $0.10(3)$ & $0.041(8)$ & $-0.415(17)$ \\
\hline
\end{tabular}

Table 6: Measured values of energy $E$, magnetization $M$, Metropolis acceptance $a_{M}$, flip acceptance $a_{f}$ and average curvature $\bar{R}$ from $N_{3}=878$ grand canonical dual simulation.

We have not yet investigated the more physically relevant case of "weak gravity" where Newton's constant is small and $\lambda_{0}$ large (at least $>0.61$ ). This would involve fixing $\lambda_{0}$ and hence $N_{0}$, then performing a canonical simulation in which $\lambda_{3}$ and $N_{3}$ are allowed to vary. Here we have instead let the system pick its preferred value of $\lambda_{0}$.

\section{Conclusions}

We have investigated the properties of the Ising model coupled to three-dimensional quantum gravity. Firstly, from micro-canonical simulations, we have determined that the dual implementation of Ising spins in the tetrahedra of the triangulation is more effective than the direct implementation on vertices, and that both cases exhibit a phase transition which is most likely second order. Then, for the dual implementation, we performed a grand canonical simulation which also displays a second order phase transition, and discovered that the effect of the Ising spins is to definitely reduce the cosmological constant and perhaps make already strong quantum gravity stronger.

The next step in these simulations is to exhaustively explore the parameter space by choosing fixed values for $\lambda_{0}$ both in the weak and strong pure quantum gravity phases, and - of course - to simulate larger systems so that finite-size scaling analysis can be done to obtain critical exponents and extrapolate to the continuum limit.

\section{Acknowledgements}

CFB was supported by DOE under contract DE-AC02-86ER40253 and by AFOSR Grant AFOSR-89-0422. I would like to thank M. E. Agishtein for help in the initial stages of this work and A. A. Migdal for useful discussions. 


\section{Figure Captions}

Fig. 1. Specific heat for direct micro-canonical simulation.

Fig. 2. Susceptibility for direct micro-canonical simulation.

Fig. 3. Specific heat for dual micro-canonical and grand canonical simulations.

Fig. 4. Susceptibility for dual micro-canonical and grand canonical simulations.

Fig. 5. Cluster size vs. diameter, at closest $\beta$ values to $\beta_{c}$, for direct $N_{0}=325$ and dual $N_{3}=878$ micro-canonical simulations, along with fits to $d$ shown as dotted and full lines respectively.

Fig. 6. Predictions for $\lambda_{0}, \lambda_{3}$ from grand canonical simulations. 


\section{References}

[1] J. Jurkiewicz, A. Krzywicki, B. Petersson and B. Soderberg, Phys. Lett. B213 (1988) 511 ;

R. Ben-Av, J. Kinar and S. Solomon, Nucl. Phys. B ( Proc. Suppl.) 20 (1991) 711;

M. E. Agishtein and C. F. Baillie, Mod. Phys. Lett. A6 (1991) 1615;

S.M. Catterall, J.B. Kogut and R.L. Renken, Phys. Rev. D45 (1992) 2957;

C. F. Baillie and D. A. Johnston, A Numerical Test of KPZ Scaling: Potts Models Coupled to Two-Dimensional Quantum Gravity, to appear in Mod. Phys. Lett. A (1992);

C. F. Baillie and D. A. Johnston, Multiple Potts Models Coupled to Two-Dimensional Quantum Gravity, to appear in Phys. Lett. B (1992).

[2] M. E. Agishtein and A.A. Migdal, Mod. Phys. Lett. A6 (1991) 1863, erratum: ibid A6 (1991) 2555;

D.V. Boulatov and A. Krzywicki, Mod. Phys. Lett. A6 (1991) 3005;

J. Ambjorn, D.V. Boulatov, A. Krzywicki and S. Varsted, Phys. Lett. B276 (1992) 432;

J. Ambjorn and S. Varsted, Three-dimensional simplicial quantum gravity, NBI-HE-9145 preprint (Sep 1991).

[3] R.H. Swendsen and J.-S. Wang, Phys. Rev. Lett. 58 (1987) 86.

[4] U. Wolff, Phys. Rev. Lett. 62 (1989) 361.

[5] C. F. Baillie, R. Gupta, K. A. Hawick and G. S. Pawley, Phys. Rev. B45 (1992) 10438.

[6] M. De Meo, D.W. Heermann and K. Binder, J. Stat. Phys. 60 (1990) 585.

[7] J.-S. Wang and D. Stauffer, Z. Phys. B78 (1990) 145.

[8] J.L. Cambier and M. Nauenberg, Phys. Rev. B34 (1986) 8071. 